\title{
Anomalous Commutator Algebra for Conformal Quantum Mechanics
}

\author{
Gino N. J. Añaños, ${ }^{1,2}$ Horacio E. Camblong, ${ }^{3}$ \\ Carlos Gorrichátegui, ${ }^{1}$ Ernesto Hernández, ${ }^{1}$ and Carlos R. Ordóñez ${ }^{1,2}$ \\ 1 Department of Physics, University of Houston, Houston, TX 77204-5506 \\ 2 World Laboratory Center for Pan-American Collaboration in Science and Technology, \\ University of Houston Center, Houston, TX 7r204-5506 \\ 3 Department of Physics, University of San Francisco, San Francisco, CA 94117-1080
}

\begin{abstract}
The structure of the commutator algebra for conformal quantum mechanics is considered. Specifically, it is shown that the emergence of a dimensional scale by renormalization implies the existence of an anomaly or quantum-mechanical symmetry breaking, which is explicitly displayed at the level of the generators of the $\mathrm{SO}(2,1)$ conformal group. Correspondingly, the associated breakdown of the conservation of the dilation and special conformal charges is derived.
\end{abstract}

PACS numbers: 11.10.Gh, 03.65.Fd, 11.30.Qc 


\section{INTRODUCTION}

The concept of symmetry plays a central role in the conceptual framework of modern physics. One of the most fruitful approaches starts by identifying a symmetry, whose actual or potential breakdown is subsequently analyzed. A particular case of this process is an anomaly - a classical symmetry that breaks down upon regularization and renormalization [1-3]. Specifically, the existence of anomalies is usually associated with the need to regularize infinities that appear in quantum-field-theory descriptions of particle and extendedobject interactions. In their continuum version, these theories require an infinite number of degrees of freedom, which in turn become the source of infinities in relevant calculations. As a consequence, regularization is unavoidable and occasionally accompanied by anomalous symmetry breaking.

In contrast with their quantum-field-theory counterparts, the concepts of regularization, renormalization, and anomalous symmetry breaking do not appear to be necessary tools in quantum mechanics. This "regular" behavior is usually ascribed to the finite number of degrees of freedom sufficient to describe these systems at low energies. However, this lore has been challenged by Jackiw [4] for the two-dimensional $\delta$-function interaction. The strongly singular nature of this potential at the origin suggests the use of regularization and renormalization as an alternative approach to quantizing the system, which would otherwise seem not to be defined. In fact, a detailed calculation for the two-dimensional $\delta$-function potential shows that the interacting system is well defined, but only after renormalization $[4$, 5]. The existence of a renormalized version of the theory and the usefulness of related fieldtheory concepts have been confirmed in a number of independent studies [6-8]. Moreover, a simple argument reveals that this interaction is scale invariant [7], but a dimensional parameter survives regularization and renormalization due to dimensional transmutation [9]. In addition, similar techniques and concepts have been used to analyze and renormalize the inverse square potential $[7,8,10-12]$, which can also be shown to be conformally invariant at the classical level $[13,14]$. These scale-invariant potentials may be regarded as the most outstanding examples of conformal quantum mechanics.

Despite our recent progress in the analysis of singular potentials in conformal quantum mechanics, the possible breakdown of their symmetry algebra at the quantum level has not yet been systematically explored. This omission is corrected in the present paper, in which 
we introduce an outline of the general theory and detailed computations for the particular case of the two-dimensional $\delta$-function interaction. The general theory is presented in Sec. II, where we review the algebraic commutator properties of the Noether charges associated with conformal invariance and show how to characterize the corresponding anomaly at the quantum level. In Sec. III we display the emergence of the conformal anomaly for

the two-dimensional $\delta$-function potential by implementing the necessary renormalization of the bound-state sector using three different regularization techniques. In Sec. IV we also show how to describe this anomalous symmetry breaking for the scattering sector of the theory. Finally, in Sec. V we present the conclusions of our work, while in the Appendix we summarize the main results on the $d$-dimensional radial Schrödinger equation that are needed throughout the paper.

\section{ANOMALOUS COMMUTATOR ALGEBRA}

In this section we consider an arbitrary scale-invariant potential $V(\mathbf{r})$. From a simple dimensional argument [7], it can be shown that the scale invariance of the action occurs if and only if $V(\mathbf{r})$ is homogeneous of degree -2 . In subsequent sections of this paper, $V(\mathbf{r})$ will be specialized to the particular case of the two-dimensional $\delta$-function interaction.

\section{A. $\mathrm{SO}(2,1)$ commutator algebra}

A straightforward analysis of the symmetries of these scale-invariant potentials under time reparametrizations shows the existence of three Noether charges. The corresponding quantum-mechanical generators are the Hamiltonian

$$
N_{1}=H \equiv \frac{p^{2}}{2 M}+V(\mathbf{r})
$$

the dilation operator

$$
N_{2}=D \equiv t H-\frac{1}{4}(\mathbf{p} \cdot \mathbf{r}+\mathbf{r} \cdot \mathbf{p})
$$

and the special conformal operator

$$
N_{3}=K \equiv t^{2} H-\frac{t}{2}(\mathbf{p} \cdot \mathbf{r}+\mathbf{r} \cdot \mathbf{p})+\frac{M}{2} r^{2},
$$

which are expected to satisfy the $\mathrm{SO}(2,1)$ Lie algebra [15]

$$
[D, H]_{\mathrm{regular}}=-i \hbar H, \quad[K, H]_{\mathrm{regular}}=-2 i \hbar D, \quad[D, K]_{\mathrm{regular}}=i \hbar K .
$$


In Eq. (4) the qualification "regular" emphasizes that the commutators follow from a naive computation in which their anomalous behavior is not explicitly considered. These scaling and commutator properties have been shown to apply to the two-dimensional $\delta$-function interaction [4] and the inverse square potential [13, 14], and are also shared by the magnetic monopole [16] and the magnetic vortex [17]. These representative examples of conformal quantum mechanics and their associated $\mathrm{SO}(2,1)$ symmetry have also been recognized in the study of a number of nonrelativistic limits of quantum field theories [18, 19]. More generally, the same basic results apply to the entire class of homogeneous potentials of degree -2 , which are both scale and conformally invariant.

The usual interpretation of scale invariance is summarized by the first commutator in Eq. (4), which shows that the scale dimension of $H$ is -1 . This scale dimension is, in fact, the "time dimension" $\mathcal{T}$ corresponding to the dimensional-analysis result $[H]=\mathcal{T}^{-1}$, in units such that $\hbar=1$ and $2 M=1$. For an arbitrary interaction, in these units, the spatial length dimension is $\mathcal{L}=\mathcal{T}^{1 / 2}$. Moreover, for the particular case of a scale-invariant theory, i.e., for a potential $V(\mathbf{r})$ homogeneous of degree -2 , the "naive algebra" and scaling $[H]=\mathcal{T}^{-1}$ are satisfied directly from the symmetry, when other subtleties are ignored [7]. That is precisely the dimensional-analysis interpretation of the first commutator in Eq. (4).

The main goal of our paper is to show the emergence of correction terms in Eq. (4) due to dimensional transmutation [9], as manifested by the presence of a dimensionful renormalization parameter $[7,8,10,11]$. In fact, this is the origin of an experimental realization of a quantum anomaly in molecular physics [20], whose underlying mechanism has also been studied within a path-integral approach [21-23].

\section{B. Anomalous commutators}

In what follows we will consider the nonperturbative definition of the Hilbert space for conformally-invariant potentials, according to the framework of Refs. [4, 7, 8, 11, 22]. This can be achieved by properly renormalizing the theory in the strong-coupling regime, with the introduction of a scale-breaking parameter. However, if the $\mathrm{SO}(2,1)$ conformal symmetry is violated upon renormalization, then the question arises as to where this symmetry breaking manifests in the commutator algebra of the operators (1)-(3). In this paper we show that 
the modification of the algebra (4) is encoded in the basic commutator

$$
[D, H]=-i \hbar H+[D, H]_{\mathrm{extra}},
$$

which acquires an "extra" piece whose expectation values will be computed below. Then, Eqs. (1)-(3) imply that

$$
[K, H]=-2 i \hbar D+2 t[D, H]_{\mathrm{extra}}, \quad[D, K]=i \hbar K-t^{2}[D, H]_{\mathrm{extra}},
$$

as follows by straightforward application of the canonical commutator relations. Thus, the extra terms in Eqs. (5) and (6) extend the commutator algebra (4) and spoil the conservation laws of the Noether charges (2) and (3), as dictated by their time evolution

$$
\frac{d A}{d t}=\frac{\partial A}{\partial t}+\frac{1}{i \hbar}[A, H]
$$

in the Heisenberg picture. In particular, the Heisenberg equations (7) imply that

$$
\frac{d D}{d t}=\frac{1}{i \hbar}[D, H]_{\mathrm{extra}}, \quad \frac{d K}{d t}=\frac{2 t}{i \hbar}[D, H]_{\mathrm{extra}} .
$$

The next step in our construction is the remarkable finding that the modified commutator algebra and the corresponding relations (8) can be evaluated in a representation-independent manner. This is achieved again by the use of the canonical commutators, which imply that

$$
[\mathbf{p} \cdot \mathbf{r}+\mathbf{r} \cdot \mathbf{p}, H]=2 i \hbar\left\{2 T-\mathcal{E}_{\mathbf{r}} V(\mathbf{r})\right\},
$$

where $T=H-V$ is the kinetic energy operator and the symbol

$$
\mathcal{E}_{\mathbf{r}}=\mathbf{r} \cdot \nabla
$$

stands for the "Eulerian derivative," which - when applied to a homogeneous functionselects the correct degree of homogeneity. The expression $\mathcal{E}_{\mathbf{r}} V(\mathbf{r})$ is a formal operator derivative that coincides with the corresponding elementary counterpart in the position representation. Then, the "anomaly operator" $\mathcal{A}(\mathbf{r})$, to be defined from the extra term $[D, H]_{\text {extra }}$ in Eq. (5), can be computed from Eqs. (2) and (9), whence

$$
\mathcal{A}(\mathbf{r}) \equiv \frac{1}{i \hbar}[D, H]_{\mathrm{extra}} \equiv \frac{1}{i \hbar}[D, H]+H=\left[\mathbb{1}+\frac{1}{2} \mathcal{E}_{\mathbf{r}}\right] V(\mathbf{r}),
$$

where $\mathbb{1}$ is the identity operator. An alternative useful expression of this anomaly (11) in $d$ dimensions is

$$
\mathcal{A}(\mathbf{r})=-\frac{1}{2}(d-2) V(\mathbf{r})+\frac{1}{2} \nabla \cdot\{\mathbf{r} V(\mathbf{r})\}
$$


Despite their deceivingly simple appearance, Eqs. (11) and (12) still fail to make the anomalous behavior explicit. This is due to the fact that the conformal anomaly can be reduced to the breakdown of the naive scaling properties of the potential. This fact is clearly displayed by Eq. (11), which shows that the dilation charge is conserved and scale invariance is maintained when $\mathcal{E}_{\mathbf{r}} V(\mathbf{r})=-2 V(\mathbf{r})$, an equation that amounts to Euler's theorem for a homogeneous potential of degree -2 . Thus, one is naively tempted to state that the conformal anomaly vanishes for the class of scale-invariant potentials. However, as we will show below, this homogeneity condition is violated: the breakdown of Euler's theorem can be traced to the singular behavior of the potential and its associated wave functions at the origin. In particular, the existence of a nonvanishing anomaly can be explicitly shown by considering the corresponding expectation values with normalized states $|\Psi\rangle$.

In subsequent sections we are going to apply these generic concepts to the two-dimensional $\delta$-function interaction. More precisely, we will show that the expressions in Eqs. (8) and (11)-(13) are indeed nontrivial due to the singular behavior of the wave function at the origin. This means that: (i) the additional term $[D, H]_{\text {extra }}$ in the commutator $[D, H]$, as defined in Eq. (5), is not identically equal to zero; (ii) relevant expectation values of this extra term $[D, H]_{\text {extra }}$ have nonzero values. Furthermore, this program can be most easily accomplished by computing the expectation value

$$
\frac{d}{d t}\langle D\rangle_{\Psi}=\langle\mathcal{A}(\mathbf{r})\rangle_{\Psi}=-\frac{1}{2}(d-2)\langle V(\mathbf{r})\rangle_{\Psi}-\frac{1}{2} \int d^{d} \mathbf{r} V(\mathbf{r}) \mathcal{E}_{\mathbf{r}}|\Psi(\mathbf{r})|^{2}
$$

in which a vanishing boundary term at infinity is dropped, after integration by parts.

\section{Properties of symmetry generators and their expectation values}

Before applying Eq. (13) and related concepts to particular potentials, we will first summarize a number of well-known albeit insightful results about quantum-mechanical expectation values. These will help interpret the values taken by the conformal anomaly within a familiar framework.

Specifically, consider a generator $A$ of a symmetry that satisfies the nontrivial condition $\partial A / \partial t \neq 0$. Let us also assume that a mechanism is provided for the existence of a state $|\Psi\rangle$ that yields a nonvanishing expectation value

$$
\left\langle\frac{\partial A}{\partial t}\right\rangle_{\Psi} \neq 0 .
$$


For the important cases considered in this paper, $A=D$ or $A=K$, this mechanism happens to be renormalization. Then, from general properties of quantum-mechanical states, the following statements can be made:

(1) If the symmetry is strictly maintained, then $-i \hbar \partial A / \partial t=[A, H] \neq 0$, so that Eq. (14) implies that $\langle[A, H]\rangle_{\Psi} \neq 0$. This is precisely what would happen with the dilation charge $D$ and conformal charge $K$, if the commutators were exactly given by Eq. (4). Reciprocally, this statement is logically equivalent to the condition that, if a state $|\Psi\rangle$ is found for which $\langle[A, H]\rangle_{\Psi}=0$, then the symmetry is necessarily broken.

(2) If there exist normalized stationary states $|\Psi\rangle$, then

$$
\langle[A, H]\rangle_{\Psi}=0
$$

is also a necessary condition. As a consequence, when Eq. (14) is satisfied, the symmetry is violated, with

$$
\frac{d}{d t}\langle A\rangle_{\Psi}=\left\langle\frac{\partial A}{\partial t}\right\rangle_{\Psi}
$$

For the dilation and conformal charges, this is only possible through the extra piece in Eq. (5), which should guarantee a subtle chain of identities

$$
i \hbar\left\langle\frac{\partial A}{\partial t}\right\rangle_{\Psi}=\left\langle[A, H]_{\text {extra }}\right\rangle_{\Psi}=-\left\langle[A, H]_{\text {regular }}\right\rangle_{\Psi}
$$

where each individual term is not zero.

In particular, the scheme discussed in point 2 above applies directly to the ground state $\left|\Psi_{(\mathrm{gs})}\right\rangle$, whenever it exists. Then, Eq. (16) [with $A=D$ defined in Eq. (2)] implies that

$$
\frac{d}{d t}\langle D\rangle_{\Psi_{(\mathrm{gs})}}=E_{(\mathrm{gs})}
$$

where $E_{(\mathrm{gs})}=\langle H\rangle_{\Psi_{(\mathrm{gs})}}$.

In the next few sections, we will verify Eq. (18) for the two-dimensional $\delta$-function interaction by an explicit computation of the anomalous correction terms. In other words, we will show that this potential exhibits a conformal quantum anomaly. 


\section{THE TWO-DIMENSIONAL $\delta$-FUNCTION INTERACTION}

In this section we will consider a two-dimensional $\delta$-function interaction

$$
V(\mathbf{r})=g \delta^{(2)}(\mathbf{r}) \equiv-\frac{\hbar^{2}}{2 M} \lambda \delta^{(2)}(\mathbf{r})
$$

In addition to defining the interaction potential, Eq. (19) introduces a dimensionless coupling $\lambda$. For the interaction (19), the conformal anomaly defined in Eq. (13), with $d=2$, is given by the formal expression

$$
\frac{d}{d t}\langle D\rangle_{\Psi}=-\frac{g}{2} \int d^{2} \mathbf{r} \delta^{(2)}(\mathbf{r}) \mathcal{E}_{\mathbf{r}}|\Psi(\mathbf{r})|^{2}
$$

As we will see next, Eq. (20) is ill defined and requires an appropriate procedure of regularization and renormalization, to be performed simultaneously with the determination of states and observables.

The conformal anomaly discussed in this paper is manifested by the existence of a nonzero value for the right-hand side of Eqs. (8), (11)-(13), and (20). For the two-dimensional deltafunction interaction, a naive argument would suggest that this time derivative in Eq. (20) is indeed identically equal to zero, because $\delta^{(2)}(\mathbf{r})$ selects a zero value in $\mathbf{r} \cdot \nabla$. However, this line of reasoning assumes that the states $|\Psi\rangle$ have a regular behavior at the origin-a condition that is explicitly violated upon renormalization in the presence of the interaction (19). More precisely, even though the regularized wave functions satisfy the regular boundary conditions (A.9) and (A.10), the renormalized wave functions acquire a logarithmic singularity at the origin. For example, the Hilbert subspace of normalized bound-states of the two-dimensional $\delta$-function interaction reduces to the one-dimensional space spanned by the renormalized ground state $[4,7,8,22]$

$$
\Psi_{(\mathrm{gs})}(\mathbf{r})=\frac{\kappa}{\sqrt{\pi}} K_{0}(\kappa r)
$$

where

$$
E_{(\mathrm{gs})}=-\frac{\hbar^{2} \kappa^{2}}{2 M}
$$

while the running coupling constant $g$ asymptotically vanishes. Specifically, the behavior of the wave function (21) near the origin is dictated by [24]

$$
K_{0}(z) \stackrel{(z \rightarrow 0)}{=}-\left[\ln \left(\frac{z}{2}\right)+\gamma\right]\left[1+O\left(z^{2}\right)\right]
$$


where $\gamma$ stands for the Euler-Mascheroni constant. Thus, the integral in Eq. (20) fails to vanish identically and confirms the purported violation of Euler's theorem. As a consequence, Eq. (20) is ill defined at the level of the renormalized quantities, but can be evaluated by going back to the regularized theory and taking the appropriate limit of its regularized counterpart.

In what follows we will regularize Eq. (20) using three distinct techniques - and each one involves defining the regularized potential, as well as the corresponding running coupling constant $\lambda$ : (i) real-space regularization with a circular-well potential; (ii) real-space regularization with a radial $\delta$-function potential; and (iii) dimensional regularization. As we will see, subtle cancellations within each one of the regularization methods combine to reproduce the same final answer (18).

\section{A. Real-space regularization with a circular-well potential}

Real-space regularization provides a scheme whereby the short-distance physics is appropriately modified for $r \lesssim a$ (where $a$ is a real-space regulator), so as to yield a well-defined problem. Of the many possible real-space regularization techniques, here it proves convenient to introduce a circular-well potential

$$
V(\mathbf{r}) \sim g \frac{\theta(a-r)}{\pi a^{2}} \equiv-\frac{\hbar^{2} \lambda}{2 M} \frac{\theta(a-r)}{\pi a^{2}},
$$

in which $\theta(\xi)$ stands for the Heaviside function.

Due to the central nature of Eq. (24), the results summarized in the Appendix can be directly applied. The corresponding Schrödinger equation for the reduced radial wave function $u_{l}(r)$ is given by

$$
\left\{\frac{d^{2}}{d r^{2}}+\left[\frac{2 M}{\hbar^{2}} E+\lambda \frac{\theta(a-r)}{\pi a^{2}}\right]-\frac{l^{2}-1 / 4}{r^{2}}\right\} u_{l}(r)=0,
$$

in which $l=|m|$, where $m$ is the usual quantum number. The bound-state solution $(E<0)$ to Eq. (25) can be written in terms of Bessel functions [24],

$$
R_{l}(r) \equiv \frac{u_{l}(r)}{\sqrt{r}}= \begin{cases}\left\{J_{l}(\tilde{k} r), N_{l}(\tilde{k} r)\right\} & \text { for } r<a \\ \left\{I_{l}(\kappa r), K_{l}(\kappa r)\right\} & \text { for } r>a\end{cases}
$$

where the curly brackets stand for linear combination, while

$$
\tilde{k}^{2}=\frac{2 M}{\hbar^{2}} E+\frac{\lambda}{\pi a^{2}}
$$


and

$$
\kappa^{2}=-\frac{2 M}{\hbar^{2}} E
$$

The regular boundary conditions at the origin (see the Appendix) and at infinity lead to the selection of $J_{l}(\tilde{k} r)$ and $K_{l}(\kappa r)$, while the continuity of the logarithmic derivative at $r=a$ provides the eigenvalue equation

$$
\tilde{k} \frac{J_{l}^{\prime}(\tilde{k} a)}{J_{l}(\tilde{k} a)}=\kappa \frac{K_{l}^{\prime}(\kappa a)}{K_{l}(k a)},
$$

in which the primes denote derivatives.

The next step is the renormalization of the system. This is implemented by finding the behavior of the running coupling constant from the consistency requirement that the eigenvalue equation (29) admit a finite ground-state energy, when $a \rightarrow 0$. As the analysis in Ref. [8] shows, this system can only sustain a bound state in the $s$ channel; this fact is confirmed by Eq. (29), which admits a nontrivial solution in the limit $a \rightarrow 0$ only for $l=0$. Correspondingly, the ground-state wave function becomes

$$
\Psi_{(\mathrm{gs})}(\mathbf{r})= \begin{cases}B J_{0}(\tilde{k} r) & \text { for } r<a \\ A K_{0}(\kappa r) & \text { for } r>a\end{cases}
$$

where the ratio between $A$ and $B$ can be determined from the additional continuity condition

$$
B J_{0}(\tilde{k} a)=A K_{0}(\kappa a)
$$

For that particular channel $(l=0)$, Eqs. $(27)$ and $(29)$, combined with the small-argument behavior of Bessel functions [in particular, Eq. (23)], provide the desired running of the coupling constant

$$
\lambda(a) \stackrel{(a \rightarrow 0)}{=}-\frac{2 \pi}{[\ln (\kappa a / 2)+\gamma]}\left\{1+O\left([\ln (\kappa a)]^{-1}\right)\right\}
$$

In Eq. (32) the hierarchy of correction terms with respect to the variable

$$
\xi=\kappa a
$$

yields the three categories $O\left([\ln \xi]^{-1}, \xi^{2} \ln \xi, \xi^{2}\right)$, including the corresponding higher orders; of these terms, the first is the dominant one. The order notation is used in Eq. (32) and thereafter, in order to keep track of all corrections with respect to small arguments and 
regularizing parameters. This procedure is suggested by the ill-defined nature of the formal expression (20), which calls for a redefinition of each factor before the limit $a \rightarrow 0$ is taken. However, it should be pointed out that the corresponding series are typically going to be convergent rather than simply asymptotic, despite our reference to asymptotic approximations.

For the computation of the anomaly (13), the values of the coefficients $A$ and $B$ should be determined. First, they are related by the condition (31), which reduces to

$$
B \stackrel{(a \rightarrow 0)}{=}-A\left[\ln \left(\frac{\kappa a}{2}\right)+\gamma\right]\left\{1+O\left([\ln (\kappa a)]^{-1}\right)\right\}
$$

Secondly, their specific asymptotic values can be explicitly obtained from the normalization condition

$$
\begin{aligned}
& 1=\int d^{2} \mathbf{r}\left|\Psi_{(\mathrm{gs})}(\mathbf{r})\right|^{2}=A^{2} 2 \pi \kappa^{-2}\left\{\mathcal{K}(\kappa a)+\left(\frac{\kappa}{\tilde{k}}\right)^{2}\left(\frac{B}{A}\right)^{2} \mathcal{J}(\tilde{k} a)\right\} \\
& \stackrel{(a \rightarrow 0)}{=} \pi \kappa^{-2} A^{2}\{1+O(\kappa a \ln [\kappa a])\},
\end{aligned}
$$

where

$$
\mathcal{K}(\xi)=\int_{\xi}^{\infty} d z z\left[K_{0}(z)\right]^{2}=\frac{1}{2}+O(\xi \ln \xi)
$$

and

$$
\mathcal{J}(\tilde{\xi})=\int_{0}^{\tilde{\xi}} d z z\left[J_{0}(z)\right]^{2}=\frac{1}{2} \tilde{\xi}^{2}\left[1+O\left(\tilde{\xi}^{2}\right)\right]
$$

with

$$
\tilde{\xi}=\tilde{k} a
$$

Thus, Eq. (35) implies that

$$
A \stackrel{(a \rightarrow 0)}{=} \frac{\kappa}{\sqrt{\pi}}\{1+O(\kappa a \ln [\kappa a])\} .
$$

Now we proceed to calculate the conformal anomaly via the regularized version of Eq. (13). For the two-dimensional $\delta$-function interaction, this is accomplished by using the regularized potential (24), in conjunction with the regularized wave function, Eqs. (30), (34), and (39), and running coupling (32). Then,

$$
\begin{aligned}
\frac{d}{d t}\langle D\rangle_{\Psi_{(\mathrm{gs})}} & =-\frac{1}{2} \int d^{2} \mathbf{r} V(\mathbf{r}) \mathcal{E}_{\mathbf{r}}\left|\Psi_{(\mathrm{gs})}(\mathbf{r})\right|^{2} \\
& =\frac{\hbar^{2}}{2 M} \frac{2 \lambda B^{2}}{\tilde{\xi}^{2}} \int_{0}^{\tilde{\xi}} d z z J_{0}(z) \mathcal{E}_{z} J_{0}(z) \\
\stackrel{(a \rightarrow 0)}{=} & E_{(\mathrm{gs})}\left\{1+O\left([\ln (\kappa a)]^{-1}\right)\right\}
\end{aligned}
$$


as follows from Eqs. (22) and (27), as well as from from the small-argument behavior of $J_{0}(z)$; in Eq. $(40), \mathcal{E}_{z}$ is the one-dimensional (radial) generalization of Eq. (10). Finally, taking the limit $a \rightarrow 0$, the conformal anomaly (40) is in perfect agreement with the expected answer (18).

\section{B. Real-space regularization with a radial $\delta$-function interaction}

An alternative real-space regularization technique is provided by a radial $\delta$-function interaction

$$
V(\mathbf{r}) \sim g \frac{\delta(r-a)}{2 \pi \alpha a} \equiv-\frac{\hbar^{2}}{2 M} \hat{\lambda} \frac{\delta(r-a)}{2 \pi a} .
$$

In addition to defining the regularized potential, Eq. (41) introduces two auxiliary quantities: (i) an arbitrary proportionality factor $\alpha$ associated with a possible ambiguity in the definition of the radial $\delta$ function [25]; (ii) a reduced coupling $\hat{\lambda}=\lambda / \alpha$. As it is to be expected, the anomaly (20), to be computed later in this section, will be independent of the undetermined "ambiguity factor" $\alpha$.

Due to the central nature of Eq. (41), the formalism of the Appendix can be directly applied again. The corresponding Schrödinger equation for the reduced radial wave function $u_{l}(r)$ is now given by

$$
\left[\frac{d^{2}}{d r^{2}}+\frac{2 M E}{\hbar^{2}}+\frac{\hat{\lambda}}{2 \pi a} \delta(r-a)-\frac{l^{2}-1 / 4}{r^{2}}\right] u_{l}(r)=0 .
$$

The bound-state solution $(E<0)$ to Eq. $(42)$, subject to the regular boundary conditions at the origin (see the Appendix) and at infinity, is given by

$$
R_{l}(r) \equiv \frac{u_{l}(r)}{\sqrt{r}}= \begin{cases}B_{l} I_{l}(\kappa r) & \text { for } r<a \\ A_{l} K_{l}(\kappa r) & \text { for } r>a\end{cases}
$$

where $\kappa$ is defined just as in Sec. III A, Eq. (28).

The eigenvalue equation follows from the condition defining the $\delta$-function discontinuity at $r=a$,

$$
\left.\frac{d u_{l}}{d r}\right|_{r=a^{+}}-\left.\frac{d u_{l}}{d r}\right|_{r=a^{-}}=-\frac{\hat{\lambda}}{2 \pi a} u_{l}(a) .
$$

Therefore, with the functions defined in Eq. (43), the eigenvalue equation takes the explicit form

$$
\kappa\left[A_{l} K_{l}^{\prime}(\kappa a)-B_{l} I_{l}^{\prime}(\kappa a)\right]=-\frac{\hat{\lambda}}{2 \pi a} A_{l} K_{l}(\kappa a)
$$


where the primes denote derivatives. A detailed analysis of Eq. (45) shows, as in Sec. III A, that a nontrivial solution exists only for $l=0$. Correspondingly, the ground-state wave function becomes

$$
\Psi_{(\mathrm{gs})}(\mathbf{r})= \begin{cases}B I_{0}(\kappa r) & \text { for } r<a \\ A K_{0}(\kappa r) & \text { for } r>a\end{cases}
$$

where $A \equiv A_{0}$ and $B \equiv B_{0}$. In addition, $A$ and $B$ can be determined from the continuity condition

$$
B I_{0}(\kappa a)=A K_{0}(\kappa a)
$$

which reduces to

$$
B \stackrel{(a \rightarrow 0)}{=}-A\left[\ln \left(\frac{\kappa a}{2}\right)+\gamma\right]\left\{1+O\left([\kappa a]^{2}\right)\right\}
$$

Moreover, the normalization condition gives

$$
1=\int d^{2} \mathbf{r}\left|\Psi_{(\mathrm{gs})}(\mathbf{r})\right|^{2}=A^{2} 2 \pi \kappa^{-2}\left\{\mathcal{K}(\kappa a)+\left(\frac{B}{A}\right)^{2} \mathcal{I}(\kappa a)\right\},
$$

where $\mathcal{K}(\xi)$ is defined in Eq. (36), while

$$
\mathcal{I}(\xi)=\int_{0}^{\xi} d z z\left[I_{0}(z)\right]^{2}=\frac{1}{2} \xi^{2}\left[1+O\left(\xi^{2}\right)\right] .
$$

Thus, Eqs. (36), (49), and (50) lead again to an expression identical to Eq. (39).

The running of the coupling constant is obtained by replacing the small-argument behavior of Bessel functions [in particular, Eq. (23)] in Eq. (45). Then,

$$
\hat{\lambda}(a) \equiv \frac{\lambda(a)}{\alpha} \stackrel{(a \rightarrow 0)}{=}-\frac{2 \pi}{[\ln (\kappa a / 2)+\gamma]}\left\{1+O\left([\kappa a]^{2} \ln [\kappa a]\right)\right\}
$$

where the correction terms with respect to the variable $\xi=\kappa a$ appear in the two categories $O\left(\xi^{2} \ln \xi, \xi^{2}\right)$, including the corresponding higher orders; of these terms, the first is the dominant one.

Finally, the conformal anomaly can be computed by replacing the regularized potential (41), the regularized wave function [Eqs. (39), (46), and (48)], and the running coupling (51) in Eq. (13). This computation yields

$$
\begin{aligned}
\frac{d}{d t}\langle D\rangle_{\Psi_{(\mathrm{gs})}} & =-\frac{1}{2} \int d^{2} \mathbf{r} V(\mathbf{r}) \mathcal{E}_{\mathbf{r}}\left|\Psi_{(\mathrm{gs})}(\mathbf{r})\right|^{2} \\
& =\frac{\hbar^{2}}{2 M} \frac{\hat{\lambda}}{2} \int_{0}^{\infty} d r \delta(r-a) \mathcal{E}_{\mathbf{r}}\left|\Psi_{(\mathrm{gs})}(\mathbf{r})\right|^{2}
\end{aligned}
$$


As the wave function (46) has a discontinuous derivative through $r=a$, the integral in Eq. (52) is conveniently computed by dividing the interval $[0, \infty)$ into the subintervals $I_{<}=$ $[0, a]$ and $I_{>}=[a, \infty)$. Then,

$$
\frac{d}{d t}\langle D\rangle_{\Psi_{(\mathrm{gs})}}=\mathcal{A}^{(<)}+\mathcal{A}^{(>)}
$$

where

$$
\mathcal{A}^{(j)}=\frac{\hbar^{2}}{2 M} \frac{\hat{\lambda}}{2} \int_{I_{j}} d r \delta(r-a) \mathcal{E}_{\mathbf{r}}\left|\Psi_{(\mathrm{gs})}^{(j)}(\mathbf{r})\right|^{2}=\frac{\hbar^{2}}{2 M} \frac{\hat{\lambda}}{2} R^{(j)}(a) \mathcal{E}_{r} R^{(j)}(a)
$$

with $j=<$ when $r<a$ and $j=>$ when $r>a$, while $\Psi_{(\mathrm{gs})}^{(j)}(\mathbf{r}) \equiv R^{(j)}(r)$ is given in Eq. (46). From Eqs. (39) and (46), as well as the small-argument behavior of Bessel functions, the exterior integral becomes

$$
\mathcal{A}^{(>)}=\frac{\hbar^{2}}{2 M} \frac{\hat{\lambda} A^{2}}{2} \xi K_{0}(\xi) K_{0}^{\prime}(\xi) \stackrel{(a \rightarrow 0)}{=} E_{(\mathrm{gs})}\left\{1+O\left(\xi^{2} \ln \xi\right)\right\}
$$

[where $\xi$ is defined in Eq. (33)], while the interior integral takes the form

$$
\mathcal{A}^{(<)}=\frac{\hbar^{2}}{2 M} \frac{\hat{\lambda} B^{2}}{2} \xi I_{0}(\xi) I_{0}^{\prime}(\xi) \stackrel{(a \rightarrow 0)}{=} E_{(\mathrm{gs})} \times O\left(\xi^{2} \ln \xi\right) ;
$$

as a result,

$$
\frac{d}{d t}\langle D\rangle_{\Psi_{(\mathrm{gs})}} \stackrel{(a \rightarrow 0)}{=} E_{(\mathrm{gs})}\left\{1+O\left([\kappa a]^{2} \ln [\kappa a]\right)\right\} .
$$

Therefore, when the limit $a \rightarrow 0$ is enforced, the conformal anomaly (40) again agrees with the predicted value, Eq. (18).

\section{Dimensional regularization}

In dimensional regularization [26] the modification of the short-distance physics is nontrivially accounted for by a dimensional generalization of the theory - the relevant physics is analytically continued from a given physical dimensionality $d_{0}$ to $d=d_{0}-\epsilon$, with $\epsilon=0^{+}$. For singular interactions, this procedure is implemented by properly extending the potential from $d_{0}$ to $d$ dimensions. Even though this generalization is somewhat arbitrary, in this paper we follow the convenient prescription provided in Refs. [7, 8, 21, 22]. Accordingly,

$$
V(\mathbf{r}) \sim g \mu^{\epsilon} \delta^{(d)}(\mathbf{r}) \equiv-\frac{\hbar^{2}}{2 M} \lambda \mu^{\epsilon} \delta^{(d)}(\mathbf{r})
$$

where the physical dimensions of the original theory are preserved by changing the dimensions of the coupling according to $g \rightarrow g \mu^{\epsilon}[7]$. 
The interaction (58) can be regarded as effectively central, so that the results of the Appendix can be applied. The corresponding Schrödinger equation for the reduced radial wave function $u_{l}(r)$, in $d \equiv 2 \nu+2=2-\epsilon$ dimensions, is given by

$$
\left[\frac{d^{2}}{d r^{2}}+\frac{2 M E}{\hbar^{2}}-\frac{(l+\nu)^{2}-1 / 4}{r^{2}}\right] u_{l}(r)=0
$$

for $r \neq 0$. Equation (59) is formally identical to that of a free particle, but it is to be supplemented by the stringent boundary condition enforced by the $\delta$-function singularity at the origin.

The bound-state solution $(E<0)$ to Eq. (59) is a linear combination of the Bessel functions $I_{l+\nu}(\kappa r)$ and $K_{l+\nu}(\kappa r)$. As usual, the boundary condition at infinity leads to the rejection of $I_{l+\nu}(\kappa r)$. As for the modified Bessel function $K_{l+\nu}(\kappa r)$, its small-argument behavior for $l \neq 0$ leads to a singular term proportional to $r^{-(l+\nu)}$. Thus, the boundary condition at the origin can only be satisfied for $l=0$, a result that agrees with the conclusions drawn from real-space regularization techniques. As a consequence, the regularized radial wave function is of the form $R(r) \propto r^{-\nu} K_{\nu}(\kappa r)$, and the corresponding normalized groundstate wave function becomes

$$
\Psi_{(\mathrm{gs})}(\mathbf{r})=\frac{\kappa}{\sqrt{\pi}} \frac{\pi^{\epsilon / 4}}{[\Gamma(1+\epsilon / 2)]^{1 / 2}} r^{\epsilon / 2} K_{-\epsilon / 2}(\kappa r) \equiv A(\epsilon) F_{\epsilon}(\kappa r),
$$

with

$$
F_{\epsilon}(z) \equiv z^{\epsilon / 2} K_{-\epsilon / 2}(z)
$$

In Eq. (60) the normalization constant $A(\epsilon)$ in $d=2(\nu+1)=2-\epsilon$ dimensions was obtained from $\int_{0}^{\infty} d z z\left[K_{p}(z)\right]^{2}=\Gamma(1+p) \Gamma(1-p) / 2$, and its asymptotic value is

$$
A(\epsilon) \stackrel{(\epsilon \rightarrow 0)}{=} \frac{\kappa}{\sqrt{\pi}}[1+O(\epsilon)] .
$$

The fact that Eq. (60) is the only bound state is a requirement of the eigenvalue equation, which follows by asking that the $\delta$-function singularity at the origin be enforced [7]. This procedure implies the condition

$$
\frac{\lambda \mu^{\epsilon}}{4 \pi}\left(\frac{2 M}{\hbar^{2}} \frac{|E|}{4 \pi}\right)^{-\epsilon / 2} \Gamma\left(\frac{\epsilon}{2}\right)=1,
$$

which displays a simple pole at $\epsilon=0$, making the theory singular for the two-dimensional unregularized case. However, for $\epsilon=0^{+}$, Eq. (63) permits the existence of the bound state $(60)$. 
Renormalization is implemented by introducing the running coupling $[7,8,22]$, which is determined in the limit $\epsilon \rightarrow 0$ from Eq. (63), i.e.,

$$
\lambda(\epsilon) \stackrel{(\epsilon \rightarrow 0)}{=} 2 \pi \epsilon\left\{1+\frac{\epsilon}{2}\left[g^{(0)}-(\ln 4 \pi-\gamma)\right]+o(\epsilon)\right\}
$$

where $\gamma$ is the Euler-Mascheroni constant and $g^{(0)}$ is an arbitrary finite part. In particular, from Eq. (64), the ground-state energy becomes

$$
E_{(\mathrm{gs})}=-\frac{\hbar^{2} \mu^{2}}{2 M} e^{g^{(0)}}
$$

Finally, the conformal anomaly can be computed by replacing the regularized potential (58) and the running coupling (64) in Eq. (13). Then,

$$
\begin{aligned}
& \frac{d}{d t}\langle D\rangle_{\Psi_{(\mathrm{gs})}}=\left[-\frac{\hbar^{2}}{2 M} \lambda(\epsilon) \mu^{\epsilon}\right]\left\{\frac{\epsilon}{2}\left\langle\delta^{(d)}(\mathbf{r})\right\rangle_{\Psi_{(\mathrm{gs})}}-\frac{1}{2} \int d^{d} \mathbf{r} \delta^{(d)}(\mathbf{r}) \mathcal{E}_{\mathbf{r}}\left|\Psi_{(\mathrm{gs})}(\mathbf{r})\right|^{2}\right\} \\
& \stackrel{(\epsilon \rightarrow 0)}{=}\left[-\frac{\hbar^{2}}{2 M}\right] \pi \epsilon\left\{\epsilon\left|\Psi_{(\mathrm{gs})}(\mathbf{0})\right|^{2}-\left[\mathcal{E}_{\mathbf{r}}\left|\Psi_{(\mathrm{gs})}(\mathbf{r})\right|^{2}\right]_{\mathbf{r}=\mathbf{0}}\right\}[1+O(\epsilon)] \\
& \stackrel{(\epsilon \rightarrow 0)}{=} E_{(\mathrm{gs})}\left\{\left[\epsilon F_{\epsilon}(0)\right]^{2}-2 \epsilon F_{\epsilon}(0) \lim _{z \rightarrow 0} \mathcal{E}_{z} F_{\epsilon}(z)\right\}[1+O(\epsilon)],
\end{aligned}
$$

where Eqs. (22) and (60) were applied in the final line, while $\mathcal{E}_{z}$ is the one-dimensional (radial) generalization of Eq. (10). The operations to be performed in Eq. (66) at the level of the regularized wave function (61) can be simplified with the use of Bessel-function identities. First, the small-argument expansion

$$
K_{p}(z) \stackrel{(z \rightarrow 0)}{=} \frac{1}{2}\left[\Gamma(p)\left(\frac{z}{2}\right)^{-p}+\Gamma(-p)\left(\frac{z}{2}\right)^{p}\right]\left[1+O\left(z^{2}\right)\right],
$$

leads to $F_{\epsilon}(0)=1 / \epsilon$, with corrections of order $O(\epsilon)$. Secondly, either from Eq. (67) again or from the identity

$$
\frac{1}{z} \frac{d}{d z}\left[z^{-p} K_{p}(z)\right]=-z^{-(p+1)} K_{p+1}(z)
$$

one concludes that $\mathcal{E}_{\xi} F_{\epsilon}(\xi)=-\xi^{\epsilon}$, with corrections of orders $O\left(\epsilon, \xi^{2}\right)$. Therefore, the final result is

$$
\begin{aligned}
\frac{d}{d t}\langle D\rangle_{\Psi_{(\mathrm{gs})}} & \stackrel{(\epsilon \rightarrow 0)}{=} E_{(\mathrm{gs})}\left\{[1+O(\epsilon)]+2 \lim _{r \rightarrow 0}(\kappa r)^{\epsilon}[1+O(\epsilon)]\right\} \\
& \stackrel{(\epsilon \rightarrow 0)}{=} E_{(\mathrm{gs})}[1+O(\epsilon)] .
\end{aligned}
$$

Remarkably, the limit $\epsilon \rightarrow 0$ should be taken only as the last step, as required by the dimensional-regularization prescription. When this procedure is properly applied, the conformal anomaly (69) again agrees with the value anticipated in Eq. (18). 


\section{SCATTERING SECTOR FOR A TWO-DIMENSIONAL $\delta$-FUNCTION IN- TERACTION}

In Sec. III we focused our analysis on the emergence of extra terms for relevant boundstate expectation values. However, a complete characterization of the interaction requires the complementary analysis in the scattering sector of the theory. For the two-dimensional $\delta$-function interaction, the compatibility of the renormalization in both sectors is well known; e.g., as discussed in Refs. $[4,7,8,22]$. In this section we now complete our analysis by using these compatibility requirements and consequently display the emergence of an anomalous commutator algebra for scattering.

Our goal is to make use of expectation values and thereby construct nonvanishing symmetry-breaking terms. Unfortunately, this construction proves to be considerably more difficult than for bound states because of the nonexistence of scattering states that are simultaneously normalized and stationary. The proper formalism to display the anomalous terms is then provided by time-dependent collision theory [27]. In addition, we conveniently switch to the Schrödinger picture as the natural way to study the time evolution of these wave packets.

Let $\Psi(\mathbf{r}, t)$ be a wave packet of positive energy evolving in $d$ dimensions from an initial state

$$
\Psi(\mathbf{r}, 0)=\int \frac{d^{d} \mathbf{q}}{(2 \pi)^{d}} \chi(\mathbf{q}) \phi_{\mathbf{q}}(\mathbf{r}),
$$

in which $\phi_{\mathbf{q}}(\mathbf{r})=\exp (i \mathbf{q} \cdot \mathbf{r})$. This function $\phi_{\mathbf{q}}(\mathbf{r})$ can be interpreted as the incident plane wave for a scattering experiment in which

$$
\psi_{\mathbf{q}}(\mathbf{r})=\phi_{\mathbf{q}}(\mathbf{r})+\frac{2 M}{\hbar^{2}} \int d^{d} \mathbf{r}^{\prime} \mathcal{G}_{d}^{(+)}\left(\mathbf{r}-\mathbf{r}^{\prime} ; q\right) V\left(\mathbf{r}^{\prime}\right) \psi_{\mathbf{q}}\left(\mathbf{r}^{\prime}\right)
$$

is the stationary-state interacting wave function for energy $E=E_{\mathbf{q}} \equiv \hbar^{2} q^{2} / 2 M$ and $q=$

$|\mathbf{q}|$, while $\mathcal{G}_{d}^{(+)}(\mathbf{R} ; q)=-i(q / 2 \pi R)^{d / 2-1} H_{d / 2-1}^{(1)}(q R) / 4$ stands for the corresponding causal Green's function.

In this section we assume that the applicability conditions and approximations of Ref. [27] are satisfied for the treatment with wave packets. In this context, the present derivation is at least sufficient to prove our claim of the existence of anomalous terms in well-defined expectation values. Then, starting with the initial condition (70), the time evolution of the 
state $|\Psi(t)\rangle$ is asymptotically described by

$$
\Psi(\mathbf{r}, t)=\int \frac{d^{d} \mathbf{q}}{(2 \pi)^{d}} \chi(\mathbf{q}) \psi_{\mathbf{q}}(\mathbf{r}) e^{-i \omega_{\mathbf{q}} t}
$$

where $\omega_{\mathbf{q}}=E_{\mathbf{q}} / \hbar$. In general, using a resolution of the form (72) between states $\left|\Psi_{1}(t)\right\rangle$ and $\left|\Psi_{2}(t)\right\rangle$, the expression for the transition matrix elements of an operator $A$ becomes

$$
\left\langle\Psi_{1}(t)|A| \Psi_{2}(t)\right\rangle=\int \frac{d^{d} \mathbf{q}^{\prime \prime}}{(2 \pi)^{d}} \int \frac{d^{d} \mathbf{q}^{\prime}}{(2 \pi)^{d}} \chi_{1}^{*}\left(\mathbf{q}^{\prime \prime}\right) \chi_{2}\left(\mathbf{q}^{\prime}\right) e^{-i\left(\omega_{\mathbf{q}^{\prime}}-\omega_{\mathbf{q}^{\prime \prime}}\right) t}\left\langle\psi_{\mathbf{q}^{\prime \prime}}|A| \psi_{\mathbf{q}^{\prime}}\right\rangle .
$$

In particular, for the expectation value of the dilation operator,

$$
\frac{d}{d t}\langle D\rangle_{\Psi(t)}=\int \frac{d^{d} \mathbf{q}^{\prime \prime}}{(2 \pi)^{d}} \int \frac{d^{d} \mathbf{q}^{\prime}}{(2 \pi)^{d}} \chi^{*}\left(\mathbf{q}^{\prime \prime}\right) \chi\left(\mathbf{q}^{\prime}\right) e^{-i\left(\omega_{\mathbf{q}^{\prime}}-\omega_{\mathbf{q}^{\prime \prime}}\right) t}\left\langle\psi_{\mathbf{q}^{\prime \prime}}\left|\frac{[D, H]_{\mathrm{extra}}}{i \hbar}\right| \psi_{\mathbf{q}^{\prime}}\right\rangle
$$

an expression which can be evaluated for a specific two-dimensional potential $V(\mathbf{r})$ from Eq. (12), whence

$$
\begin{aligned}
\left\langle\psi_{1}\left|\frac{[D, H]_{\text {extra }}}{i \hbar}\right| \psi_{2}\right\rangle & =\frac{1}{2} \int d^{2} \mathbf{r} \psi_{1}^{*}(\mathbf{r}) \nabla \cdot(\mathbf{r} V(\mathbf{r})) \psi_{2}(\mathbf{r}) \\
& =-\frac{1}{2} \int d^{2} \mathbf{r} V(\mathbf{r}) \mathcal{E}_{\mathbf{r}}\left[\psi_{1}^{*}(\mathbf{r}) \psi_{2}(\mathbf{r})\right]
\end{aligned}
$$

Therefore, for the two-dimensional $\delta$-function interaction,

$$
\begin{aligned}
\left\langle\psi_{\mathbf{q}^{\prime \prime}}\left|\frac{[D, H]_{\text {extra }}}{i \hbar}\right| \psi_{\mathbf{q}^{\prime}}\right\rangle & =-\frac{g}{2}\left\{\psi_{\mathbf{q}^{\prime \prime}}^{*}(0)\left[\hat{\mathcal{E}}_{\mathbf{r}} \psi_{\mathbf{q}^{\prime}}(\mathbf{r})\right]_{\mathbf{r}=0}+\left[\hat{\mathcal{E}}_{\mathbf{r}} \psi_{\mathbf{q}^{\prime \prime}}^{*}(\mathbf{r})\right]_{\mathbf{r}=0} \psi_{\mathbf{q}^{\prime}}(0)\right\} \\
& =\left(-\frac{\hbar^{2}}{2 M}\right) 4 \sqrt{q^{\prime \prime} q^{\prime}} f_{q^{\prime \prime}}^{*}\left(\mathbf{\Omega}^{(2)}\right) f_{q^{\prime}}\left(\mathbf{\Omega}^{(2)}\right),
\end{aligned}
$$

where

$$
f_{q} \equiv f_{q}\left(\mathbf{\Omega}^{(2)}\right)=\sqrt{\frac{2 \pi}{q}}\left[\ln \left(\frac{E_{\mathbf{q}}}{\left|E_{(\mathrm{gs})}\right|}\right)-i \pi\right]^{-1}
$$

is the isotropic two-dimensional scattering amplitude [22], in which the angular dependence $\mathbf{\Omega}^{(2)}$ can be omitted. Finally, from Eqs. (74) and (76),

$$
\frac{d}{d t}\langle D\rangle_{\Psi(t)}=\left(-\frac{2 \hbar^{2}}{M}\right) \int \frac{d^{2} \mathbf{q}^{\prime \prime}}{(2 \pi)^{2}} \int \frac{d^{2} \mathbf{q}^{\prime}}{(2 \pi)^{2}} \chi^{*}\left(\mathbf{q}^{\prime \prime}\right) \chi\left(\mathbf{q}^{\prime}\right) e^{-i\left(\omega_{\mathbf{q}^{\prime}}-\omega_{\mathbf{q}^{\prime \prime}}\right) t} \sqrt{q^{\prime \prime} q^{\prime}} f_{q^{\prime \prime}}^{*}\left(\mathbf{\Omega}^{(2)}\right) f_{q^{\prime}}\left(\mathbf{\Omega}^{(2)}\right),
$$

whence it follows that

$$
\frac{d}{d t}\langle D\rangle_{\Psi(t)}=-\frac{\hbar^{2}}{2 M}|\mathcal{F}(t)|^{2}
$$

where

$$
\mathcal{F}(t)=2 \int \frac{d^{2} \mathbf{q}}{(2 \pi)^{2}} \chi(\mathbf{q}) e^{-i \omega_{\mathbf{q}} t} \sqrt{q} f_{q}
$$


Equations (79) and (80) can be verified in the three regularization schemes introduced earlier and constitute the main result of this section. Just as the logarithmic behavior of Eqs. (21) and (23) was the source of the nontrivial anomalous commutator in the bound state sector, the singular behavior $H_{0}^{(1)}(z) \stackrel{(z \rightarrow 0)}{=} 1+2 i[\ln (z / 2)+\gamma] / \pi\left[\right.$ up to terms $\left.O\left(z^{2}\right)\right]$ in Eq. (71) yields the nontrivial expression (79). This final result admits the following interpretation: a wave packet undergoes a time evolution dictated by the linear superposition of its initial Fourier components; as the scattering amplitude depends upon a scale $\left|E_{(g s)}\right|$, the ensuing symmetry charges are no longer conserved.

\section{CONCLUSIONS}

In conclusion, we have explicitly shown the anomalous nature of the commutator algebra in conformal quantum mechanics for the two-dimensional $\delta$-function interaction. These results are supported by detailed computations performed with three distinct regularization techniques in both the bound-state sector and the scattering sector of the interacting theory.

The crucial point in this anomalous behavior is that extra terms in the commutators of the $\mathrm{SO}(2,1)$ generators arise from the dimensionally-transmuted scale of the renormalized theory. The implication of the existence of these nonvanishing terms at the level of nonconserved symmetry charges was explored and general properties of quantum-mechanical averages were used to shed light on the physical meaning of our results.

A similar but considerably subtler analysis can be applied to the inverse square potential in any number of dimensions; the details of this procedure will be discussed elsewhere. Incidentally, an alternative technique that has been widely used to deal with singular potentials is the method of self-adjoint extensions [4]. It would be interesting to investigate the same issues using that method and to provide a comparison with our results [28].

\section{Acknowledgments}

This research was supported in part by an Advanced Research Grant from the Texas Higher Education Coordinating Board and by the University of San Francisco Faculty Development Fund. One of us (G.N.J.A.) gratefully acknowledges the generous support from the World Laboratory. We also thank Professor Roman Jackiw, Professor Luis N. Epele, 
Professor Huner Fanchiotti, and Professor Carlos A. García Canal for stimulating discussions.

\section{APPENDIX: CENTRAL POTENTIALS IN d DIMENSIONS}

In this appendix, we summarize a few basic results for the $d$-dimensional radial Schrödinger equation. We only state those properties that are needed for the applications of the general theory discussed in this paper. In particular, these results are essential for the proper use of dimensional regularization, even though the particular problems analyzed herein are strictly two-dimensional.

Conservation of $d$-dimensional angular momentum permits the separation of the radial coordinate $r$ from the angular variables $\Omega^{(d)}$. Moreover, it leads to an angular dependence proportional to the hyperspherical harmonics $Y_{l m}\left(\Omega^{(d)}\right)$. The associated wave function $\Psi(\mathbf{r})=Y_{l m}\left(\Omega^{(d)}\right) R_{l}(r)$ includes a radial piece $R_{l}(r)$, which depends upon the quantum number $l$ as well as the energy $E$. Furthermore, $R_{l}(r)$ satisfies the differential equation

$$
\left\{\Delta_{r}^{(d)}-\frac{l(l+d-2)}{r^{2}}+\frac{2 M}{\hbar^{2}}[E-V(r)]\right\} R_{l}(r)=0,
$$

in which the radial Laplacian is given by

$$
\Delta_{r}^{(d)}=\frac{1}{r^{d-1}} \frac{\partial}{\partial r}\left(r^{d-1} \frac{\partial}{\partial r}\right)=\frac{1}{r^{(d-1) / 2}} \frac{\partial^{2}}{\partial r^{2}}\left[r^{(d-1) / 2}\right]-\frac{(d-1)(d-3)}{4 r^{2}} .
$$

Then, it proves convenient to define the reduced radial wave function

$$
u_{l}(r)=R_{l}(r) r^{(d-1) / 2},
$$

which satisfies an effective one-dimensional Schrödinger equation

$$
\left\{\frac{d^{2}}{d r^{2}}+\frac{2 M}{\hbar^{2}}[E-V(r)]-\frac{\Lambda_{l, d}}{r^{2}}\right\} u_{l}(r)=0,
$$

in which the angular-momentum effective potential has a coupling constant

$$
\Lambda_{l, d}=l(l+d-2)+(d-1)(d-3) / 4=(l+\nu)^{2}-1 / 4,
$$

with

$$
\nu=\frac{d}{2}-1
$$


For the interactions discussed in this paper, Eq. (A.4) provides a direct transition to a differential equation of the form

$$
\left[\frac{d^{2}}{d r^{2}}+\left(k^{2}-\frac{p^{2}-1 / 4}{r^{2}}\right)\right] u(r)=0,
$$

whose solution $u(r)=\sqrt{r} Z_{p}(k r)$ is given in terms of Bessel functions $Z_{p}(k r)$ of order $p$.

Furthermore, Eq. (A.4) should be supplemented by appropriate boundary conditions at the origin and at infinity. First, asymptotically with respect to $r \rightarrow \infty$, the boundstate solutions should have a zero limit in order to satisfy the square-integrability condition. Likewise, the scattering solutions are subject to the usual requirement that the wave function asymptotically reproduce the incident wave plus an outgoing scattered state [7]. On the other hand, the boundary condition at $r=0$ is much subtler and requires additional study.

It turns out that the boundary condition at the origin is the key factor that determines the nature of the singularity at the origin. As such, it is used to establish the classification of potentials into the regular and singular families. In this framework, both the regular and the regularized singular interactions are subject to the limiting condition

$$
r^{2} V(r) \stackrel{r \rightarrow 0}{\longrightarrow} 0
$$

In particular, asymptotically with respect to $r \rightarrow 0$, the wave function is reduced to the solution of the radial part of Laplace's equation. As a consequence, the regular boundary condition becomes

$$
R_{l}(r) \propto r^{l}
$$

which is usually restated in terms of the weaker condition

$$
u_{l}(0)=0
$$

[1] J. S. Bell and R. Jackiw, Nuovo Cimento A 60, 47 (1969); S. Adler, Phys. Rev. 177, 2426 (1969); S. Adler and W. A. Bardeen, ibid. 182, 1517 (1969).

[2] S. B. Treiman, R. Jackiw, B. Zumino, and E. Witten, Current Algebras and Anomalies (World Scientific, Singapore, 1985).

[3] R. Jackiw, in Quantum Mechanics of Fundamental Systems, edited by C. Teitelboim (Plenum, New York, 1988). 
[4] R. Jackiw, in M. A. B. Bég Memorial Volume, edited by A. Ali and P. Hoodbhoy (World Scientific, Singapore, 1991).

[5] An earlier renormalization treatment of the $\delta$-function interaction can be found in C. Thorn, Phys. Rev. D 19, 639 (1979). See K. Huang, Quarks, Leptons, and Gauge Fields, 2nd. ed. (World Scientific, Singapore, 1992), Sec. 10.8.

[6] B. Holstein, Am. J. Phys. 61, 142 (1993); C. Manuel and R. Tarrach, Phys. Lett. B 328, 113 (1994); S. K. Adhikari and T. Frederico, Phys. Rev. Lett. 74, 4572 (1995); and references therein.

[7] H. E. Camblong, L. N. Epele, H. Fanchiotti, and C. A. García Canal, Ann. Phys. (N.Y.) 287, 14 (2001).

[8] H. E. Camblong, L. N. Epele, H. Fanchiotti, and C. A. García Canal, Ann. Phys. (N.Y.) 287, $57(2001)$.

[9] S. Coleman and E. Weinberg, Phys. Rev. D 7, 1888 (1973).

[10] K. S. Gupta and S. G. Rajeev, Phys. Rev. D 48, 5940 (1993).

[11] H. E. Camblong, L. N. Epele, H. Fanchiotti, and C. A. García Canal, Phys. Rev. Lett. 85, $1590(2000)$.

[12] S. R. Beane et al., Phys. Rev. A 64, 042103 (2001).

[13] R. Jackiw, Phys. Today 25(1), 23 (1972).

[14] V. de Alfaro, S. Fubini, and G. Furlan, Nuovo Cimento A 34, 569 (1976).

[15] B. Wybourne, Classical Groups for Physicists (Wiley, New York, 1974).

[16] R. Jackiw, Ann. Phys. (N.Y.) 129, 183 (1980).

[17] R. Jackiw, Ann. Phys. (N.Y.) 201, 83 (1990).

[18] R. Jackiw and S. Y. Pi, Phys. Rev. D 42, 3500 (1990).

[19] O. Bergman, Phys. Rev. D 46, 5474 (1992); O. Bergman and G. Lozano, Ann. Phys. (N.Y.) 229, 416 (1994).

[20] H. E. Camblong, L. N. Epele, H. Fanchiotti, and C. A. García Canal, Phys. Rev. Lett. 87, $220402(2001)$.

[21] H. E. Camblong and C. R. Ordóñez, hep-th/0109003.

[22] H. E. Camblong and C. R. Ordóñez, Phys. Rev. A 65, 052123 (2002).

[23] H. E. Camblong and C. R. Ordóñez, Mod. Phys. Lett. A 17, 817 (2002).

[24] Handbook of Mathematical Functions, edited by M. Abramowitz and I. A. Stegun (Dover, New 
York, 1972).

[25] The value $\alpha=1$ is the obvious choice when one starts with the "circular" $\delta$ function: $\delta(r-a)$, with $a \neq 0$. In a sense, this is operationally a weak two-sided radial $\delta$ function. However, if one started from the unregularized problem (with $a=0$ ), the value of $\alpha$ would be undetermined, with $\alpha=1$ and $\alpha=1 / 2$ being the usual "strong" definitions of the radial $\delta$ function; see G. Barton, Elements of Green's Functions and Propagation (Oxford University Press, Oxford, 1989), p. 11, and Sec. 1.4.2.

[26] C. G. Bollini and J. J. Giambiagi, Nuovo Cimento Soc. Ital. Fis. B 12, 20 (1972); G. 't Hooft and M. Veltman, Nucl. Phys. B44, 189 (1972); J. F. Ashmore, Lett. Nuovo Cimento Soc. Ital. Fis. 4, 289 (1972).

[27] K. Gottfried, Quantum Mechanics, Volume I: Fundamentals (Addison-Wesley, Reading, MA, 1966), Sec. 12.2 .

[28] After completion of our work we learned of the following paper that uses the method of self-adjoint extensions to analize the conformal anomaly of the two-dimensional $\delta$-function potential: J. G. Esteve, Phys. Rev. D 66, 125013 (2002). 